\title{
INFLUÊNCIA DE FAIXAS DE VEGETAÇÃO NATIVA EM POVOAMENTOS DE Eucalyptus cloeziana SOBRE POPULAÇÃO DE Oxydia vesulia (LEPIDOPTERA: GEOMETRIDAE) ${ }^{1}$
}

\author{
Germi Porto Santos ${ }^{2}$, Teresinha Vinha Zanuncio ${ }^{3}$, Eloisio Vinha ${ }^{4}$ e José Cola Zanuncio ${ }^{5}$
}

\begin{abstract}
RESUMO - O eucalipto é a espécie florestal mais plantada no Brasil, e por pertencer à família Myrtaceae sofre ataques de insetos que migram de hospedeiros nativos dessa planta, entre os quais Oxydia vesulia (Lepidoptera: Geometridae), espécie responsável por danos significativos a plantas desse grupo, em várias regiões do País. O estabelecimento e a preservação de reservas nativas têm sido uma das alternativas para reduzir a ação de agentes daninhos, pois promovem o aumento da diversidade ecológica e, conseqüentemente, favorecem a multiplicação e diversidade de inimigos naturais. Neste estudo, a coleta dos Lepidoptera foi realizada em duas situações, ou seja, em povoamentos de eucalipto sem e com faixas de vegetação nativa, utilizando armadilhas luminosas, dispostas em cinco diferentes situações de composição vegetal: 1 - no interior de uma área de cerrado a $100 \mathrm{~m}$ de sua borda; 2 - na zona de contato entre o cerrado e o eucaliptal; 3 - no interior do eucaliptal a $250 \mathrm{~m}$ de sua borda; 4 - no centro da faixa de cerrado, portanto a aproximadamente $500 \mathrm{~m}$ da borda; e 5 - no interior do eucaliptal a $750 \mathrm{~m}$ de sua borda. $O$. vesulia apresentou 329 indivíduos, sendo mais abundante no sistema sem faixas, com 266 indivíduos, e apenas 63 indivíduos no sistema com faixas. Essa espécie foi constante $(51,43 \%)$ no sistema sem faixas e acessória (45,71\%) no com faixas, com freqüência de 1,15 e 1,66\%, respectivamente, com coleta de maior número de indivíduos dessa espécie a partir da segunda quinzena de fevereiro, em ambos os sistemas. No sistema com faixas, $O$. vesulia foi mais coletada no interior do eucaliptal a $250 \mathrm{~m}$ da borda, com 31 indivíduos, enquanto no sem faixas o maior número de indivíduos dessa espécie foi registrado no interior do eucaliptal a $750 \mathrm{~m}$ de sua borda.
\end{abstract}

Palavras-chave: Lagartas-desfolhadoras; Eucalyptus spp. e fragmento florestal.

\section{INFLUENCE OF NATIVE VEGETATION STRIPS ON PLANTATIONS OF Eucalyptus cloeziana ON POPULATION OF Oxydia vesulia (LEPIDOPTERA: GEOMETRIDAE)}

\begin{abstract}
Species of the eucalypt genus represent the majority of forest plantations in Brazil. Because these species belong to the Myrtaceae family, they have been attacked by insects of native host plants of this family including Oxydia vesulia (Lepidoptera: Geometridae), which is responsible for significant damage to eucalyptus plants in many areas in Brazil. The establishment and preservation of native reserves have been one of the alternatives used aiming to reduce the effect of harmful agents since it increases ecological diversity, consequently favouring the multiplication and diversity of natural enemies. Lepidoptera species were collected in two eucalyptus plantations, one with and the other without strips of native vegetation with light traps, in five plant situations: 1-inside a savannah area at 100 meters from its border; 2- in the contact area between the savannah and the eucalyptus plantation; 3- inside the eucalyptus plantation at 250 meters from its border; 4- in the center of a savannah strip at approximately 500 meters from the border of the savannah area and 5-inside the eucalyptus plantation at 750 meters from the border of the savannah area. A total of 329 individuals of $\boldsymbol{O}$. vesulia was collected with this species being more abundant in the area without strips with 266 individuals, while only
\end{abstract}

1 Recebido para publicação em 6.6.2001.

Aceito para publicação em 20.6.2002.

2 Pesquisador, EMBRAPA/EPAMIG-CTZM/DBA-UFV, Caixa Postal 216, 36570-000 Viçosa-MG, <germi@mail.ufv.br>;

${ }^{3}$ Professor Substituto, Departamento de Biologia Animal da Universidade Federal de Viçosa - UFV, 36571-000 Viçosa-MG;

${ }^{4}$ Bolsista de Aperfeiçoamento do CNPq; ${ }^{5}$ Professor Titular, Departamento de Biologia Animal/BIOAGRO, UFV. 
63 individuals were collected in the area with strips. This species was constant (51.43\%) in the system without strips and accessory (45.71\%) in the area with strips while its frequency was 1.15 and $1.66 \%$, respectively, in these systems with higher number of individuals from the second fortnight of February in both systems. $\boldsymbol{O}$. vesulia showed a higher number of individuals in the system with strips, specially inside the eucalyptus plantation at 250 meters from the border of the savannah area, with 31 individuals. The system without strips showed the highest number of individuals inside the eucalyptus plantation at 750 meters from the border of the savanah area.

Key words: Defoliating caterpillars, Eucalyptus spp. and forest fragment.

\section{INTRODUÇÃO}

No Brasil, as espécies do gênero Eucalyptus ocupam área plantada de cerca de 2.954 .780 ha, e sua introdução em maior escala surgiu com a Lei dos Incentivos Fiscais, em 1965 (STCP/INDUFOR, 1998). Tal fato se deve ao seu rápido crescimento, à aplicabilidade de sua madeira para diversos fins, à sua habilidade em desenvolver-se em vasta variedade de habitats e ao fácil manejo. Esses reflorestamentos proporcionam importantes benefícios, como o de diminuir a pressão exaustiva sobre reservas nativas. Contudo, o incremento dessas áreas provoca problemas ambientais e interfere em ecossistemas naturais. Uma grave conseqüência é o crescente aumento de espécies de insetos que passaram a se adaptar a essa cultura, propiciado pela farta oferta de alimento e pela diminuição da diversidade e abundância de seus inimigos naturais (Almeida et al., 1987), provocado pela simplificação do ambiente natural.

Dentre as espécies plantadas para fins energéticos, o Eucalyptus cloeziana vem se destacando como uma das mais importantes, principalmente por sua boa densidade e seus altos teores de carbono fixo, requisito imprescindível para este fim (Vital \& Della Lucia, 1986). Esta espécie é uma das mais adequadas para regiões de cerrado, com grande potencial para reflorestamento, mas é também mais suscetível aos ataques de lagartas-desfolhadoras (Oliveira et al., 1984).

A fauna de uma árvore pode ser influenciada por fatores como estrutura morfológica e composição química das folhas, arquitetura da planta, relações espécies/área e reservas de espécies nativas (Moore et al., 1991). O estabelecimento e a manutenção dessas reservas são fundamentais para o controle biológico natural de insetos fitófagos em florestas implantadas, especialmente em regiões de cerrado, onde o sub-bosque, um fator importante no equilíbrio das populações de insetos no eucaliptal, é pouco desenvolvido. Por isto, em várias regiões do cerrado de Minas Gerais a preservação de áreas naturais de vegetação nativa e faixas dessa vegetação, intercaladas aos plantios de eucalipto, tem sido uma das ações adotadas por empresas reflorestadoras, visando preservar o estoque de inimigos naturais como predadores e parasitóides.

A ocorrência de espécies do gênero Oxydia (Lepidoptera: Geometridae) em plantios de eucalipto no Brasil foi registrada por Berti Filho (1981), em Minas Gerais e São Paulo, e por Santos et al. (1986), em Minas Gerais, em surtos endêmicos. Em maio de 1985 Oxydia vesulia foi detectada em caráter epidêmico, em uma área de 250 ha de E. cloeziana, no município de Alagoinhas, Bahia (Santos et al., 1993). Na realidade, esta espécie ocorre em diversas partes do Brasil. Espindola \& Gonçalves (2000) relatam sua presença na região de Jacareí, São Paulo, sem mencionarem a magnitude de danos.

O objetivo deste trabalho foi avaliar a influência de faixas de vegetação nativa que ligam fragmentos florestais e entre talhões de eucalipto sobre populações de O. vesulia (Cramer, 1779), em área de cerrado, visando estudar a existência de um gradiente de diversidade desse inseto no eucaliptal em relação ao cerrado e as diferenças entre plantios de eucalipto com ou sem faixas de vegetação nativa, por meio da análise faunística e da classificação das comunidades.

\section{MATERIAL E MÉTODOS}

O experimento foi conduzido em duas localidades, conforme o sistema avaliado.

\subsection{Sistema com Faixas}

$\mathrm{Na}$ fazenda Galheiros, em Paineiras, Estado de Minas Gerais, na $\mathrm{V} \& \mathrm{M}$ Tubes, em plantio de E. cloeziana F. Muell. com espaçamento de $3 \times 2,5 \mathrm{~m}$, 
com sub-bosque ralo e divisas com cerrado e outros talhões de eucalipto. A altitude média desta área é de $600 \mathrm{~m}$, com clima subtropical úmido e solo Latossolo Vermelho-Escuro distrófico (Golfari et al., 1978). A temperatura média anual é de $22,5^{\circ} \mathrm{C}$, com médias das mínimas e das máximas, respectivamente, de 16,5 e $28,8^{\circ} \mathrm{C}$, e a precipitação pluviométrica média anual de 1.518,2 mm (Mara, 1992). Neste sistema de plantio, a cada $500 \mathrm{~m}$ de reflorestamento são mantidas faixas de cerrado de $25 \mathrm{~m}$ de largura, ligadas entre si e a reservas ou ilhas de vegetação nativa, com $400 \mathrm{~m}$ a cada 10 ha. Além disto, as áreas que margeam cursos d'água, lagoas, represas e veredas são consideradas de preservação permanente.

Adultos de $O$. vesulia foram coletados com cinco armadilhas luminosas, distantes $250 \mathrm{~m}$ uma da outra, nos seguintes pontos: ponto 1 - interior de uma área de cerrado a $100 \mathrm{~m}$ da borda; 2 - zona de contato entre o cerrado e o eucaliptal; 3 - interior do eucaliptal a $250 \mathrm{~m}$ da borda; 4 - centro da faixa de cerrado, portanto a aproximadamente $500 \mathrm{~m}$ da borda; e 5 - interior do eucaliptal a $750 \mathrm{~m}$ de sua borda. Este último ponto estava localizado a $450 \mathrm{~m}$ de outra reserva de vegetação nativa, situada fora da área da empresa. As zonas de contato entre o cerrado e o eucaliptal e entre os talhões de eucalipto correspondiam a estradas de terra com, aproximadamente, $7 \mathrm{~m}$ de largura.

\subsection{Sistema sem Faixas}

Na fazenda Itapoã, em Paraopeba, Estado de Minas Gerais, na V \& M Tubes, em plantios de E. cloeziana, com espaçamento $3 \times 2,5 \mathrm{~m}$, com sub-bosque ralo e divisas com plantios de eucalipto. A altitude média da área é de $700 \mathrm{~m}$, clima subtropical úmido e solo Latossolo Vermelho-Escuro distrófico (Golfari et al., 1978). A temperatura média anual é de $22,1{ }^{\circ} \mathrm{C}$, com médias das mínimas e das máximas, respectivamente, de 16,4 e $29,2{ }^{\circ} \mathrm{C}$, e a precipitação pluviométrica média anual de $1.230 \mathrm{~mm}$ (Mara, 1992). A localização dos pontos de coleta em relação à borda do plantio de eucalipto foi igual àquela do sistema com faixas. A única diferença foi que no sistema sem faixas o ponto 4 localizou-se na zona de contato entre dois talhões de eucalipto.

Nos dois plantios de eucalipto, a coleta dos lepidópteros foi feita com cinco armadilhas luminosas, dispostas em ziguezague, visando aumentar a distância e minimizar o efeito da possível interferência entre elas. As coletas foram efetuadas de outubro de 1993 a abril de 1994, com três amostragens por ponto, a cada 15 dias. Essas armadilhas foram do tipo Intral Al-12 com luz negra, alimentadas por baterias de 12 volts e colocadas a $2 \mathrm{~m}$ do solo, considerando-se o ponto médio do comprimento da lâmpada.

Os insetos foram coletados em sacos plásticos, acoplados ao funil da armadilha, contendo tiras de papeljornal e um frasco com acetado de etila e um pavio, para acelerar a morte e evitar o descamamento excessivo dos insetos (Ferreira \& Martins, 1982). As armadilhas eram ligadas entre 18 e 19 horas e desligadas no dia seguinte, entre 7 e 8 horas, quando o material coletado era recolhido e levado ao Centro de Apoio à Pesquisa e Experimentação Florestal (CAPEF), para separação dos adultos de $O$. vesulia, que foram montados, sendo alguns deles incorporados à coleção do Laboratório de Entomologia da Universidade Federal de Viçosa, em Viçosa, Estado de Minas Gerais.

Foram calculadas a freqüência e a constância de O.vesulia nos dois sistemas, pela fórmula de Dajoz (1973):

- Índice de freqüência: $I F=\frac{N}{T} \times 100$

em que IF = índice de freqüência; $\mathrm{N}=$ número total de indivíduos de cada espécie; e $\mathrm{T}=$ número total de indivíduos de todas as espécies.

- Índice de constância: $C=\frac{P}{N} \times 100$

em que $\mathrm{C}$ = índice de constância; $\mathrm{P}=$ número de coletas em que a espécie foi capturada; e $\mathrm{N}$ = número total de coletas.

Para obter o índice de freqüência, foi adotada a classificação de Bodenheimer (1955): espécie constante $(\mathrm{X})$ - presente em mais de $50 \%$ das coletas; espécie acessória $(\mathrm{Y})$ - presente em 25 a 50\% das coletas; e espécie acidental (Z) - presente em menos de $25 \%$ das coletas.

\section{RESULTADOS E DISCUSSÃO}

O. vesulia foi constante, com $51,43 \%$ no sistema sem faixas, e acessória, com $45,71 \%$ no com faixas, com freqüência de 1,15 e $1,66 \%$, respectivamente. $O$ fato de $O$. vesulia ter sido constante no sistema sem faixas pode ser devido à sua maior adaptação à monocultura de eucalipto, além de desses plantios de eucalipto serem

R. Árvore, Viçosa-MG, v.26, n.4, p.499-504, 2002 
mais antigos e extensos, com poucas áreas de cerrado. Deve-se ressaltar ainda que o número de espécies de uma comunidade é determinado, entre outros fatores, pelo tempo em que ela está disponível para colonização pelos colonizadores potenciais (Price, 1984), entre os quais se enquadrariam as espécies acessórias. Fraser \& Lawton (1994) citam que, do conjunto de espécies nativas, as polífagas têm maior potencial de colonização do hospedeiro introduzido, porém tal fato não significa, necessariamente, o abandono de seu hospedeiro nativo. A freqüência semelhante apresentada por $O$. vesulia, nos dois sistemas, concorda com Brown (1984), que mostrou que a freqüência das espécies em determinado local exibe um padrão regular, com muitas espécies relativamente raras e restritas e algumas abundantes e amplamente distribuídas. Estas últimas apresentam, em geral, maiores flutuações populacionais em determinadas regiões e são capazes de explorar maior quantidade de recursos que as primeiras (Brown, 1984; Gaston \& Lawton, 1988).

Foram obtidos 329 indivíduos de $O$. vesulia, sendo estes mais abundantes no sistema sem faixas, com 266 indivíduos, contra 63 indivíduos no com faixas (Figura 1). $\mathrm{O}$ maior número de indivíduos foi coletado, em ambos os sistemas, a partir da segunda quinzena de fevereiro (Quadro 1). O fato de O. vesulia ter apresentado menor número de indivíduos no sistema com faixas pode ser devido a este tipo de plantio apresentar maior diversidade de vegetação, com maior riqueza e menor abundância de indivíduos de uma mesma espécie, incluindo os inimigos naturais, especialmente os generalistas, formando comunidades mais estáveis que as monoculturas (Risch et al., 1983; Altieri \& Letourneau, 1984; Sheehan, 1986; Andow, 1991).

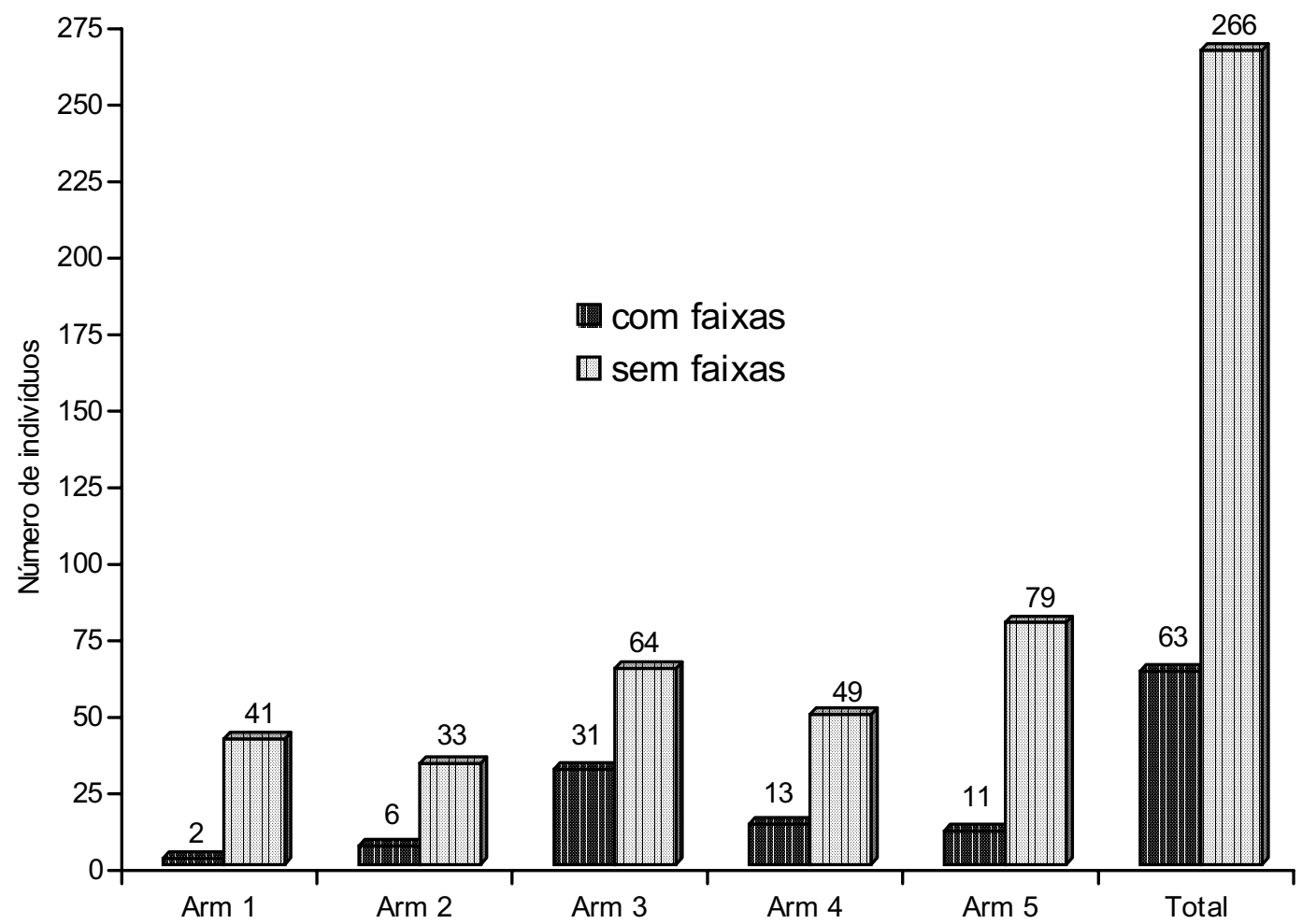

Figura 1 - Número de adultos de Oxydia vesulia (Lepidoptera: Geometridae) coletados por armadilha em plantios de Eucalyptus cloeziana com (Paineiras) e sem (Paraopeba) faixas de vegetação nativa. Minas Gerais, Brasil. Outubro de 1993 a abril de 1994.

Figure 1 - Number of adults of Oxydia vesulia (Lepidoptera: Geometridae), collected per light trap in a plantation of Eucalyptus cloeziana with (Paineiras) and without (Paraopeba) strips of native vegetation, from. October 1993 to April 1994, Minas Gerais, Brazil. 
Quadro 1 - Número de adultos de Oxydia vesulia (Lepidoptera: Geometridae) coletados por armadilha em plantios de Eucalyptus cloeziana com (Paineiras) e sem (Paraopeba) faixas de vegetação nativa. Minas Gerais, Brasil. Outubro de 1993 a abril de 1994

Table 1 - Number of adults of Oxydia vesulia (Lepidoptera: Geometridae), collected per light trap in a plantation of Eucalyptus cloeziana with (Paineiras) and without (Paraopeba) strips of native vegetation, from. October 1993 to April 1994, Minas Gerais, Brazil

\begin{tabular}{|c|c|c|c|c|c|c|c|c|c|c|c|c|c|c|}
\hline \multirow{2}{*}{ Ponto de Coletas } & \multicolumn{2}{|c|}{$\overline{\text { Outubro }}$} & \multicolumn{2}{|c|}{ Novembro } & \multicolumn{2}{|c|}{ Dezembro } & \multicolumn{2}{|c|}{ Janeiro } & \multicolumn{2}{|c|}{ Fevereiro } & \multicolumn{2}{|c|}{ Março } & \multicolumn{2}{|c|}{ Abril } \\
\hline & $\mathrm{CF}^{*}$ & $\mathrm{SF}^{* *}$ & $\mathrm{CF}$ & SF & $\mathrm{CF}$ & $\mathrm{SF}$ & $\mathrm{CF}$ & SF & $\mathrm{CF}$ & SF & $\mathrm{CF}$ & SF & $\mathrm{CF}$ & SF \\
\hline Cerrado & 0,00 & 0,50 & 0,00 & 0,00 & 0,00 & 0,00 & 0,00 & 0,00 & 0,00 & 0,00 & 4,60 & 0,00 & 3,00 & 0,25 \\
\hline Borda & 0,00 & 1,50 & 0,00 & 0,00 & 0,00 & 0,00 & 0,20 & 0,00 & 0,67 & 0,00 & 4,20 & 0,50 & 1,50 & 1,00 \\
\hline Eucalipto & 0,00 & 2,50 & 0,00 & 0,00 & 0,00 & 0,00 & 0,40 & 0,00 & 1,00 & 1,33 & 6,60 & 0,75 & 4,33 & 6,00 \\
\hline Transição & 0,00 & 3,50 & 0,00 & 0,00 & 0,17 & 0,00 & 0,20 & 0,00 & 2,67 & 0,00 & 5,60 & 1,75 & 1,83 & 1,50 \\
\hline Eucalipto & 0,00 & 4,50 & 0,00 & 0,00 & 0,00 & 0,00 & 0,00 & 0,00 & 3,00 & 0,33 & 7,20 & 0,50 & 5,67 & 2,00 \\
\hline
\end{tabular}

$* \mathrm{CF}=$ com faixa $\mathrm{e}^{* * \mathrm{SF}}=$ sem faixa.

Além de o sistema sem faixas ter apresentado maior número de indivíduos de $O$. vesulia que o com faixas, a distribuição de abundância dessas espécies nos diferentes pontos de coleta foi mais homogênea (Figura 1). No sistema sem faixas, o número de indivíduos nas armadilhas 5 (79) e 3 (64), localizadas nos talhões de eucalipto, foi maior que naquelas dos pontos de transição eucalipto/ eucalipto (49), centro do cerrado (41) e na zona de contato entre o cerrado e o eucaliptal (33) (Figura 1). No sistema sem faixas, o número de indivíduos de $O$. vesulia no cerrado (41) e na borda (33) foi maior que o do sistema com faixas (63) (Figura 1), realçando o fato do benefício proporcionado pelas faixas nativas. Segundo Price (1984), isto acontece devido à maior quantidade de recursos deste tipo de sistema, o que permite que os insetos colonizem e se reproduzam mais rapidamente. A coleta de maior número de indivíduos no interior do eucaliptal reforça a tese de que a homogeneidade do ambiente e, principalmente, a maior disponibilidade de alimento facilitam o estabelecimento de espécies presentes na área, conseqüentemente elas proliferam mais facilmente. Soma-se a isto o fato de que ambientes mais homogêneos dificultam o estabelecimento de inimigos naturais, maiores responsáveis pelo controle biológico de insetos-praga (Risch, 1981; Andow \& Risch, 1985; Andow, 1991).

O sistema com faixas apresentou menor número de indivíduos de $O$. vesulia no cerrado (2) e na borda (6) (Figura 1), os quais podem ser caracterizados como comunidades distintas. Esses habitats possuem maior diversificação e complexidade estrutural de vegetação, o que, teoricamente, os tornariam mais pobres em número de indivíduos dessa espécie. O maior número de indivíduos de $O$. vesulia na transição eucalipto/eucalipto decorreu, provavelmente, do fato de esta armadilha estar localizada em local mais aberto, o que permite que sua luz atinja maiores distâncias e atraia insetos presentes no eucalipto e, também, aqueles que ocasionalmente circulem no seu raio de ação. Segundo Strong Jr. (1979), estes últimos totalizam $91,45 \%$ das espécies presentes em pequenas ilhas oceânicas, sendo coletados com pouca freqüência e não persistindo nos locais de coleta.

Os monocultivos propiciam o surgimento e a proliferação de surtos de insetos daninhos com maior freqüência e intensidade, especialmente em povoamentos florestais puros e com idade semelhante (Altieri \& Letourneau, 1984). Todavia, devido à tendência atual de preservar parte dos remanescentes florestais nativos e outros recursos naturais, aliada à criação de corredores naturais, entremeando povoamentos florestais e ligando fragmentos nativos isolados, podem ser esperados menores problemas com agentes daninhos, principalmente lepidópteros-desfolhadores. Esta iniciativa permite a diversificação do estrato vegetal, tornando o ecossistema mais heterogêneo, o que propiciará a proliferação e a manutenção de inimigos naturais. Sugere-se como complementação deste estudo a avaliação do impacto dos inimigos naturais sobre as populações de insetos, nos dois sistemas.

\section{AGRADECIMENTO}

À Fundação de Amparo à Pesquisa do Estado de Minas Gerais (FAPEMIG) e ao Conselho Nacional de Desenvolvimento Científico e Tecnológico (CNPq), pelas bolsas concedidas a autores deste trabalho, e à $\mathrm{V} \& \mathrm{M}$ Tubes, pelos recursos disponibilizados no decorrer deste estudo. 


\section{REFERÊNCIAS BIBLIOGRÁFICAS}

ALMEIDA, A. F.; LARANJEIRO, A. J.; LEITE, J. E. M. O melhoramento ambiental no manejo integrado de pragas: um exemplo na Aracruz florestal. Silvicultura, v. 39, p. 21-25, 1987.

ALTIERI, M. A.; LETOURNEAU, D. K. Vegetation diversity and insect pest outbreaks. CRC Critical Review Plant Science, v. 2, p. 131-169, 1984.

ANDOW, D. A. Vegetation diversity and arthropod population response. Annual Review Entomology, v. 36, p. 561-586, 1991

ANDOW, D. A.; RISCH, S. J. Predation in diversified agroecosystems: relations between a coccinellid predator and its food. Journal of Applied Ecology, v. 22, p. 357$372,1985$.

BERTI FILHO, E. Insetos associados a plantações de espécies do gênero Eucalyptus em estados da Bahia, Espírito Santo, Mato Grosso do Sul, Minas Gerais e São Paulo. Piracicaba: Escola Superior de Agricultura "Luiz de Queiroz", 1981. Tese (Livre-Docente) - Escola Superior de Agricultura Luiz de Queiroz, 1981.

BODENHEIMER, F. S. Precis d'ecologie animale. Paris: Payot., 1955. $315 \mathrm{p}$.

BROWN, J. H. On the relationship between abundance and distribution of species. American Naturalist, v. 124, p. 255-279, 1984.

DAJOZ, R. Ecologia geral. Petrópolis: Vozes, 1973. $472 \mathrm{p}$.

ESPINDOLA, C. B.; GONÇALVES, L. Biologia de Oxydia vesulia (Cramer, 1779) (Lepidoptera: Geometridae).

Floresta e Ambiente, v. 7, n. 1, p. 80-87, 2000.

FERREIRA, P. S. F.; MARTINS, D. S. Contribuição ao método de captura de insetos por meio de armadilha luminosa, para a obtenção de exemplares sem danos morfológicos. Revista Ceres, v. 9, n. 165, p. 538-543, 1982.

FRASER, S. M.; LAWTON, J. H. Host range expansion by British moths onto introduced conifers. Ecology of Entomology, v. 19, p. 127-137, 1994.

GASTON, K. J.; LAWTON, J. H. Patterns in the distribution and abundance of insect populations. Nature, v. 331, p. 709-712, 1988.

R. Árvore, Viçosa-MG, v.26, n. 4, p.499-504, 2002
GOLFARI, L.; CASER, R. L.; MOURA, V. P. G. Zoneamento ecológico esquemático para reflorestamento no Brasil. Belo Horizonte: 1978. 66 p. PRODEPEF, PNUD/FAO/IBDF/BRA-45. (Série Técnica, 11).

BRASIL. Ministério da Agricultura e Reforma Agrária. Normas Climatológicas (1961-1990). Nacional de Meteorologia. Brasília: DF, 1992, 84 p.

MOORE, R.; WARRINGTON, S.; WHITTAKER, J. B. Herbivory by insects on oak trees in pure stands compared with paired mixtures. Journal of Applied Ecology, v. 28, p. 290-304. 1991.

OLIVEIRA, A. C. et al. Resistência interespecífica de Eucalyptus spp. (Myrtacea) à lagarta desfolhadora Thyrinteina arnobia Stoll, 1782 (Lepidoptera: Geometridae). Revista Árvore, v. 8, n. 2, p. 93-103, 1984.

PRICE, P. W. Insect ecology. New York: John Wiley \& Sons, 1984. 607 p.

RISCH, S. J. Insect herbivore abundance in tropical monocultures and polycultures: an experimental test of two hypotheses. Ecology, v. 62, n. 5, p. 1325-1340, 1981.

RISCH, S. J.; ANDOW, D.; ALTIERI, M. A.

Agroecosystem diversity and pest control. Data, tentative conclusions and new research directions. Environment Entomologyst, v. 12, p. 625-629, 1983.

SANTOS, G. P.; ANJOS N.; ALVES, A. P.; ZANUNCIO, J. C. Bionomia de Oxydia vesulia (Cramer, 1779) (Lepidoptera; Geometridae), desfolhador de eucalipto. Revista Árvore, v. 10, n. 2, p. 161-167, 1986.

SANTOS, G. P. et al. Descrição das lagartas desfolhadoras. In: ZANUNCIO, J. C. (Coord.). Manual de pragas em florestas. Lepidoptera desfolhadores de eucalipto: biologia, ecologia e controle. Viçosa: Folha de Viçosa, 1993. p. 12-66.

SHEEHAN, W. Response by specialist and generalist natural enemies to agroecosystem diversification: a selective review. Environment Entomology, v. 15, p. 456-461, 1986.

STCP/INDUFOR. Brazilian forest products Industry into the $21^{\text {st }}$ century. Curitiba: 1998.

STRONG Jr., D. R. Biogeographic dynamics of insect-host plant communities. Annual Review of Entomology, v. 24, p. 89-119, 1979.

VITAL, B. R.; DELLA LUCIA, R. M. Propriedades físicas e mecânicas da madeira de eucalipto. Informe Agropecuário, v. 12, n. 141, p. 71-74, 1986. 